\title{
Insuficiência renal crônica como fator de risco para insuficiência cardíaca
}

\author{
Chronic renal failure as a risk fator for heart failure
}

La insuficiencia renal crônica como fator de riesgo de insuficiencia cardiaca

\author{
Ana Lidia de Campos Lico \\ ORCID: https://orcid.org/0000-0002-0272-4315 \\ Instituto Tocantinense Antônio Carlos SA, Brasil \\ E-mail: analidiacamposlico@gmail.com \\ Leticia Freitas \\ ORCID: https://orcid.org/0000-0001-7963-6770 \\ Instituto Tocantinense Antônio Carlos SA, Brasil \\ E-mail: leticia_freitas090@ @otmail.com \\ Ana Luiza Barbosa de Oliveira Cerqueira \\ ORCID: https://orcid.org/0000-0003-2931-281X \\ Instituto Tocantinense Antônio Carlos SA, Brasil \\ E-mail: analuiza_519@hotmail.com \\ Julia Mendes Barbosa \\ ORCID: https://orcid.org/0000-0002-5814-2840 \\ Instituto Tocantinense Antônio Carlos SA, Brasil \\ E-mail: juliammendes@icloud.com \\ Ana Claudia Garcia Rosa \\ ORCID https://orcid.org/0000-0002-8566-3174 \\ Instituto Tocantinense Antônio Carlos SA, Brasil \\ E-mail: anaclaudiagarcia @uft.edu.br
}

\begin{abstract}
Resumo
O sistema renal e o sistema cardíaco estão intimamente ligados, ou seja, caso ocorra uma alteração nesses sistemas, essa alteração influi diretamente e/ou indiretamente no funcionamento do outro. Dentre as alterações mais comuns, relacionadas a esses dois sistemas, está a insuficiência renal crônica (IRC) e a insuficiência cardíaca (IC). A IRC é uma condição em que os rins perdem a sua capacidade de funcionamento. Já a IC é uma síndrome clínica caracterizada pela incapacidade do coração de funcionar adequadamente como bomba, ou seja, há alteração no déficit de contração e relaxamento, sendo este incapaz de enviar sangue para o restante do corpo. Este trabalho tem por objetivo realizar uma revisão integrativa de literatura para verificar a relação entre IRC e IC. Para o levantamento dos artigos na literatura, foi realizada uma busca nas seguintes bases de dados: Literatura Latino-Americana e do Caribe em Ciências da Saúde (LILACS), Medical Literature Analysis and Retrieval Sistem on-line (Medline), Scielo, Cochrane e Up to Date. Foram utilizados, os seguintes descritores e suas combinações nas línguas portuguesa, inglesa e espanhola: "Insuficiência Renal Crônica", "Insuficiência Cardíaca", "Doença renal crônica e doença cardíaca" e "Doença renal crônica relacionada à doença cardíaca". A análise dos estudos da literatura demonstrou que insuficiência renal crônica se transforma em um fator de risco para a insuficiência cardíaca dependendo de fatores predisponentes, como: anemia por deficiência de ferro, arteriosclerose, hipertensão, lesão renal prévia, etc. Dessa forma, essa relação afirma que a IRC é um fator de risco para IC.
\end{abstract}

Palavras-chave: Insuficiência cardíaca; Doença renal crônica; Revisão de literatura.

\begin{abstract}
The renal system and the cardiac system are closely connected, in other words, if there's a change in one of these systems, this alteration directly and/or indirectly influences the functioning of the other. Among the most common changes, related to these two systems, are chronic kidney disease (CKD) and heart failure (HF). CKD is a condition where the kidneys lose their ability to function. HF, on the other hand, is a clinical syndrome characterized by the heart's inability to function properly as a pump, that's, there's a modification in both the contraction deficit and it's relaxation, which is unable to send blood to the rest of the body. This work aims to carry out an integrative literature review to verify the relationship between $\mathrm{CKD}$ and HF. For the survey of articles in the literature, a search was carried out in the following databases: Latin American and Caribbean Literature in Health Sciences (LILACS), Medical Literature Analysis and Retrieval Sistem online (Medline), Sicelo, Cochrane and Up to Date. The following descriptors and their combinations in Portuguese, English and Spanish languages were used to search for articles: "Chronic Kidney Failure", "Heart Failure", "Chronic Kidney Disease and Heart Disease" and "Chronic Kidney Disease Related to Heart Disease". The analysis of studies in the literatute showed that chronic kidney diseade becomes a risk factor for heart failure depending on predisposing factor, such as: iron deficiency anemia,
\end{abstract}


arteriosclerosis, hipertension, previous kidney demage, etc. Thus, this relationship affirms that CKF is a risk factor for HF.

Keywords: Heart failure; Chronic kidney disease; Literature review.

\section{Resumen}

El sistema renal y el sistema cardíaco están estrechamente vinculados, es decir, si hay una alteración en estos sistemas, esta alteración influye directa y / o indirectamente en el funcionamiento del otro. Entre las alteraciones más comunes relacionadas con estos dos sistemas se encuentran la insuficiencia renal crónica (IRC) y la insuficiencia cardíaca (IC). El IRC es una afección en la que los riñones pierden su capacidad para funcionar. La IC, es un síndrome clínico caracterizado por la incapacidad del corazón para funcionar correctamente como bomba, es decir, existe una alteración en el déficit de contracción y relajación, que es incapaz de enviar sangre al resto del cuerpo. Este trabajo tiene como objetivo realizar una revisión integradora de la literatura para verificar la relación entre IRC e IC. Para el relevamiento de artículos en la literatura se realizó una búsqueda en las siguientes bases de datos: Literatura Latinoamericana y del Caribe en Ciencias de la Salud (LILACS), Medical Literature Analysis and Retrieval Sistem on-line (Medline), Scielo, Cochrane e Up to Date. Se utilizaron los siguientes descriptores y sus combinaciones en portugués, inglés y español: "Insuficiencia renal crónica", "Insuficiencia cardíaca", "Enfermedad renal crónica y cardiopatía" y "Enfermedad renal crónica relacionada con enfermedades cardíacas". El análisis de estudios en la literatura mostró que la insuficiencia renal crónica se convierte en un factor de riesgo de insuficiencia cardíaca en función de factores predisponentes, como: anemia ferropénica, arteriosclerosis, hipertensión, daño renal previo, etc. Así, esta relación afirma que el IRC es un factor de riesgo de IC.

Palabras clave: Insuficiencia cardíaca; Enfermedad renal crónica; Revisión de literatura.

\section{Introdução}

A insuficiência renal crônica (IRC) é uma condição em que os rins perdem a sua capacidade de funcionamento, ou seja, de efetuar as suas funções básicas como por exemplo: eliminação de substâncias tóxicas, manter o equilíbrio de eletrólitos no corpo humano, filtrar o sangue e dentre outras. Segundo Kumar et al. (2013), “a doença renal crônica é resultado de progressivas fibroses geradas por qualquer tipo de lesão renal (...) Esses eventos resultam em estágio renal final, em que os túbulos glomerulares, interstício e vasos apresentam-se esclerosados, independentemente do foco primário da lesão”. Esse tipo de perda da função renal é lenta, progressiva e irreversível. De acordo com Ammirati e Canziani (2009), dentro da população dos portadores de IRC, sabe-se que a principal causa de mortalidade é a doença cardiovascular, sendo a insuficiência cardíaca (IC) a mais comum.

Segundo Kumar et al (2013) “A Insuficiência cardíaca congestiva (ICC) surge quando o coração não consegue produzir débito suficiente para satisfazer as demandas metabólicas dos tecidos ou apenas consegue satisfazê-las sob pressões de enchimento superiores às normais (...) Na ICC, o coração em falência não consegue mais bombear de modo eficiente o sangue levado até ele pela circulação venosa. O resultado é o aumento do volume ventricular diastólico final, que provoca elevação das pressões diastólicas finais e, por fim, das pressões venosas também”.

Nesse contexto, entende-se que a IC é uma síndrome clínica caracterizada pela incapacidade do coração de funcionar adequadamente como bomba, ou seja, há alteração tanto no déficit de contração quanto no seu relaxamento, sendo incapaz de enviar sangue para o restante do corpo. Sendo assim, compromete o funcionamento de todo o organismo.

Por ser uma complicação rotineira da IRC, a IC faz com que a fração de ejeção seja reduzida, complicando, assim, a filtração sanguínea. Pelo fato de os dois sistemas estarem intimamente ligados, a fim de promover a homeostase através do sistema renina-angiotensina-aldosterona, o início fisiopatológico das complicações se dá pela desregulação do referido mecanismo compensatório, provocando alterações como hipertrofia ventricular, redução da capacidade vasodilatadora e disfunção endotelial (Vasan et al., 1999).

Segundo Canziani (2004), as patologias cardiovasculares são a principal causa de óbito em pacientes com doença renal crônica. Dessa forma, o presente trabalho busca realizar uma revisão integrativa da literatura com a finalidade de auxiliar os profissionais da área da saúde e a população em geral, que buscam por mais informações de como a insuficiência renal crônica pode atuar como fator de risco para insuficiência cardíaca. 


\section{Metodologia}

O presente artigo trata-se de uma revisão integrativa da literatura de natureza básica, qualitativa, sem o intuito de aplicar em campo, de caráter exploratório, de maneira que envolve levantamento de estudos pregressos e análise de exemplos. Portanto, se trata de uma documentação indireta de característica bibliográficas ou fontes secundárias, que busca revisar a relação entre insuficiência renal crônica e insuficiência cardíaca.

Trata-se de um estudo com coleta de dados realizado a partir de fontes secundárias, por meio de levantamento bibliográfico, com objetivo de compreender como a Insuficiência Renal Crônica (IRC) se torna um fator de risco para insuficiência cardíaca (IC). Para o levantamento dos artigos na literatura, foram realizadas buscas nas seguintes bases de dados: Literatura Latino-Americana e do Caribe em Ciências da Saúde (LILACS), Medical Literature Analysis and Retrieval Sistem on-line (Medline), Scielo, Cochrane e Up to Date, sendo os artigos publicados no período de 1999 a 2021.

Foram utilizados, para busca dos artigos, os seguintes descritores e suas combinações nas línguas portuguesa, inglesa e espanhola: "Insuficiência Renal Crônica", "Insuficiência Cardíaca", "Doença renal crônica e doença cardíaca" e "Doença renal crônica relacionada à doença cardíaca". Os critérios de inclusão definidos para a seleção dos artigos foram: artigos publicados em português, inglês e espanhol; artigos na íntegra que retratam a temática referente aos temas de pesquisa e artigos publicados e indexados nos referidos bancos de dados nos últimos vinte anos.

Durante a coleta de dados, primeiramente foi realizada uma leitura seletiva, sendo selecionados os artigos que se tratam dos temas: Insuficiência Renal Crônica, Insuficiência Cardíaca e a Insuficiência Renal Crônica como fator de risco para doença cardiovascular. Em um segundo momento, foi realizada uma leitura crítica dos artigos selecionados, nos quais foram analisados outros fatores de risco que poderiam levar ao acometimento cardiovascular.

Para extrair os dados dos artigos selecionados, foi utilizado um instrumento previamente elaborado capaz de assegurar que a totalidade dos dados relevantes seja extraída, a fim de minimizar o risco de erros na transcrição, garantir precisão na checagem das informações e servir como registro, conforme Souza et al. (2010).

A análise dos dados efetuou-se através de uma listagem dos fatores que associam a insuficiência renal crônica à insuficiência cardíaca, descritas nos trabalhos científicos pesquisados. Posteriormente, os dados obtidos foram interpretados e comparados. Durante o processo de comparação dos dados, foram destacados os fatores que obtiveram maior número de menções que relacionaram as patologias supracitadas. A análise dos estudos selecionados, quanto ao delineamento de pesquisa e quanto à síntese dos dados extraídos dos artigos, foi realizada de forma descritiva, possibilitando observar, contar, descrever e classificar os dados, com o intuito de reunir o conhecimento produzido sobre o tema explorado na revisão.

No total, foram selecionados 42 artigos sobre o tema, sendo que, desses, dois foram excluídos por não atenderem aos critérios de inclusão. Dessa forma, os resultados apresentados a seguir são decorrentes da análise de 40 artigos encontrados nas bases e idiomas supracitados.

\section{Resultados e Discussão}

A análise dos estudos da literatura demonstrou que a insuficiência renal crônica se transforma em um fator de risco para a insuficiência cardíaca dependendo de fatores predisponentes.

Segundo Dummer et al. (2007), os fatores que associam IRC E ICC são múltiplos, podendo ser classificados em fatores tradicionais e não tradicionais. Tradicionais são os fatores de risco com elevada prevalência, como hipertensão arterial, dislipidemia, diabetes mellitus e tabagismo. Já os fatores ditos não tradicionais são tais como inflamação, estresse oxidativo, infecção persistente, proteinúria e hiperfosfatemia.

Abaixo, são enumerados os principais fatores que associam a IRC e a IC: 


\subsection{Anemia por deficiência de ferro}

Dentre os fatores que relacionam a doença renal crônica e insuficiência cardíaca, podemos destacar a deficiência de ferro. O ferro é importante nos processos metabólicos celulares, sendo assim, sua carência afeta diretamente os miócitos, levando a disfunção contrátil do miocárdio, contribuindo para a progressão da insuficiência cardíaca (Kumar et al, 2013).

Guimarães e Ferreira (2010), descreve a anemia como consequência da perda da produção de eritropoietina devido à falência renal, levando a uma retenção de líquidos e um aumento do volume plasmático, bem como uma diminuição da sobrevida eritrocitária pelo aumento da concentração de ureia.

Portanto, associando essa vertente com a cascata da inflamação decorrente da doença renal crônica, explicada em Abensur (2010), onde o estado de inflamação eleva a produção da enzima hepcidina pelo fígado que resulta na inibição da absorção de ferro no duodeno e a mobilização de ferro da sua reserva pelas células do sistema reticuloendotelial, temos a clara ligação entre a evolução da DRC com a susceptibilidade aos quadros de ICC.

De acordo com Amenós, et al. (2017), apesar de aumentar o risco de morbimortalidade, a anemia ferropriva é um fator de grande poder modificável no tratamento da insuficiência cardíaca.

Ammirati e Canziani (2009), também associam a anemia ao desenvolvimento de hipertrofia ventricular esquerda, podendo ser o efeito precipitante de lesões cardiovasculares.

\subsection{Lesão renal prévia}

Uma lesão renal progressiva, segundo Abboud (2010), primeiramente ocorre de maneira silenciosa, em que o poder adaptativo do rim consegue mascarar patologias, dependendo da lesão e áreas afetadas. Um exemplo é a hiperfiltração glomerular, um mecanismo adaptativo, em que a taxa de filtração é aumentada a fim de manter a homeostasia corporal, porém, uma consequência disso é a proteinúria, que acarreta em um desenvolvimento de uma futura insuficiência renal.

Tendo em vista que esse mecanismo hiperfiltrativo é um fator importante para o desenvolvimento da IRC, como diz Denic et al. (2017), sabe-se que associada a comorbidades como obesidade e doenças renais prévias, essa taxa de filtração glomerular é aumentada. Consequentemente, relacionada a doenças cardiovasculares, como o aumento da pressão arterial, algumas medidas protetoras para diminuir a TFG conseguem reduzir a velocidade da formação de cicatrizes irreversíveis no rim, como o uso de terapias anti-hipertensivas, principalmente inibidor de da enzima conversora de angiotensina (ECA) ou um bloqueador do receptor da angiotensina II (Perkovic et al., 2019).

Essa associação confirma a ideia do coração e do rim funcionarem juntos não só como uma bomba-filtro, e sim como um mecanismo primário complexo, capaz de retirar a homeostase corporal ao perder a interconectividade entre eles (Martins et al., 2011).

\subsection{Aterosclerose}

A aterosclerose é um dos principais fatores que associam as duas situações clínicas abordadas. Em pacientes com doença renal crônica, podemos observar um aumento de citocinas pró-inflamatórias, descrito por Pecoits-Filho (2002):

"Níveis circulantes elevados de citocinas pró-inflamatórias e proteínas da fase aguda de resposta inflamatória são encontrados com frequência em pacientes com insuficiência renal crônica, o que demonstra um estado de inflamação crônica. A inflamação está envolvida nos processos de desnutrição e aterosclerose, ambos comuns nos pacientes portadores de IRC, e essa correlação pode decorrer de vários mecanismos. As causas de inflamação crônica são multifatoriais, podendo refletir comorbidades presentes nos pacientes ou representar fator causal para o desenvolvimento de doença cardiovascular.".

As citocinas atuam acelerando o processo de aterogênese, levando a disfunção endotelial e obstrução de vasos sanguíneos, causando um aumento significativo no risco de doença cardiovascular. 


\subsection{Hipertensão arterial}

Segundo Bortolotto (2008), a hipertensão arterial se encontra como o principal desencadeador da IRC, não excluindo a possibilidade de a mesma ser consequência da IRC; deste modo fica evidente que a associação entre essas afecções eleva consideravelmente o risco cardiovascular.

Ainda de acordo com Bortolotto (2008), a etiologia da hipertensão tem causas variáveis, porém, quando observada em pacientes com insuficiência renal crônica, os principais mecanismos fisiopatológicos evidenciados são: elevação da concentração sérica salina e do volume sanguíneo, bem como a hiperatividade do sistema renina-angiotensina-aldosterona (SRAA) e disfunção endotelial.

Segundo Kusumota et al., (2004), em casos de lesões renais graves os rins deixam de eliminar escorias nitrogenadas e ainda não conseguem regular o balanço de água e sódio, provocando a retenção de líquidos e o surgimento de hipertensão arterial e edema, podendo levar a graves consequências.

Para Bortolotto (2008), nos casos de hipertensão arterial associada a insuficiência renal o tratamento corre de maneira mais intensa visando metas pressóricas menores e tendo por base de tratamento farmacológico os anti-hipertensivos diuréticos. Ademais, são indispensáveis as mudanças nos hábitos de vida, sendo o objetivo de todo esse conjunto de ações retardar a evolução da doença renal nos estágios iniciais e reduzir o risco cardiovascular em todos os estágios.

\subsection{Hipertrofia ventricular esquerda}

Segundo Kumar et al. (2013), o coração tem a função de distribuir sangue para o restante do nosso organismo, dessa forma, alguma alteração nesse músculo acarreta em consequências fisiológicas. Sua câmara mais importante é o ventrículo esquerdo (VE), pois ele além de receber sangue do átrio esquerdo (AE), bombeia sangue oxigenado para todo o corpo. Assim, alterações dessa câmara, como a hipertrofia ventricular esquerda (HVE), causam danos irreparáveis ao corpo.

Ainda de acordo, Kumar et al. (2013), descreve que a HVE é uma resposta adaptativa do músculo cardíaco da parede do VE ao aumento do esforço realizado pelo coração, ou seja, há um aumento de tamanho dessa câmara. O VE sofre alterações como o seu endurecimento e a perda de elasticidade, o que impede o seu funcionamento adequado fazendo assim com que o coração produza uma força maior de contração para o bombeamento do sangue.

Essa é uma das patologias mais comuns nos pacientes com doenças cardiovasculares, acarretando diversas consequências. Em um estudo, foi constatado que dentre os inscritos na pesquisa, metade dos pacientes hospitalizados por insuficiência cardíaca (IC) apresentam a função sistólica do ventrículo esquerdo (FSVE) conservada. De acordo com Dizeo, et al. (2012), tal estudo também mostrou que a deterioração da FSVE ocorreu em 52\% dos pacientes, que foi considerado o limite da fração de ejeção inferior a $45 \%$.

Relacionando essa patologia com a doença renal crônica (DRC), em uma literatura de Ammirati e Canziani (2009), um estudo foi realizado e $90 \%$ dos pacientes com DRC apresentam HVE. Além de constatar que a HVE é a alteração cardiovascular mais frequentemente observada em pacientes com DRC, e sua prevalência e severidade aumentam à medida que diminui a função renal.

Além dessas complicações citadas acima, essa alteração do músculo cardíaco pode causar a morte, pois a HVE está associada a prognóstico desfavorável, sendo que mais de 2/3 dos pacientes em diálise no estudo realizado por Bucharles et al. (2010), morreram por insuficiência cardíaca congestiva ou morte súbita.

\subsection{Disturbios minerais}

Segundo Truyst et al. (2021), outro ponto relevante, são os distúrbios minerais que o pacientes com Doença Renal Crônica (DRC) pode apresentar, esses por sua vez, possuem uma forte relação naqueles que fazem a Diálise Peritoneal como 
tratamento alcançando cerca de metade dos casos de óbito e um terço das hospitalizações nesses pacientes. Anormalidades de cálcio sérico, fosforo, paratormônio, vitamina $\mathrm{D}$, anormalidades na remodelação óssea e calcificação extraóssea ajudam para aumentar a morbidade e susceptivelmente para prognósticos indesejáveis nesses pacientes.

De acordo com Lucca, et al. (2011), o cálcio realiza papéis fisiológicos significativos no metabolismo humano e está presente como a constituição do esqueleto, nos componentes entre as células e no citosol celular, e a manutenção dos níveis séricos adequados de cálcio é importante para a manutenção da homeostase. Alterações de cálcio metabólico podem gerar problemas nas glândulas paratireoide, câncer e lesões ósseas.

Para Porto et al. (2016), no paciente renal crônico, a quantidade de fósforo é modificada devido à perda de células renais, resultando a baixa excreção de fosforo, culminando em hiperfosfatemia. Essa retenção pode funcionar inibindo a produção de vitamina D ativa, pela inibição da enzima 1-a-hidroxilase renal, responsável pela conversão da vitamina D para a forma ativa. Outro evento que acompanha é a hipocalcemia, causada pela baixa absorção intestinal desse mineral. Os baixos níveis de cálcio estimulam a maior produção de PTH. "Em estudos epidemiológicos feitos com grande número de pacientes portadores de DRC, observa-se que até $80 \%$ dos mesmos apresentam valores de PTH acima dos valores laboratoriais normais de referência, especialmente aqueles que se encontram em programa regular de terapia renal substitutiva (hemodiálise e diálise peritoneal).

Ainda para Porto et al. (2016), como agravo maior, pacientes dialíticos de longa data exibem retenção de fósforo acentuada aumentando o cálcio e fósforo acima do nível crítico, podendo gerar calcificações ectópicas, sobretudo vasculares. Estas alterações podem evoluir para graves eventos como obstrução coronariana e desordens cardiovasculares.

\subsection{Disfunção diastólica}

Segundo Bastos et al. (2020), a disfunção diastólica (DD) pode se agravar para um Insuficiência Cardíaca, conhecida como insuficiência cardíaca com fração de ejeção preservada, situação que vem antes da disfunção sistólica. Na Disfunção Diastólica a maioria dos pacientes apresenta hipertrofia ventricular esquerda por conta da sobrecarga aumento de volume e hipertensão durante o tratamento com a Diálise.

Ainda para Bastos et al. (2020), para se explicar essa incidência da Disfunção Diastólica se incluem a rigidez aórtica (Calcificação vascular) e aumento volêmico que causa uma sobrecarga de volume, que são rotineiramente observadas na DRC, em especial em estágios mais avançados.

\section{Conclusão}

Entre os principais envolvidos na associação entre IRC e IC estão: anemia por deficiência de ferro, na qual a interferência está ligada aos miócitos que perdem parte da sua função, contribuindo para a IC; aterosclerose que pode ser provoca pelo aumento de citocinas pro-infamatórias que estão aumentadas na DRC, além de outras doenças cardiovasculares, como a hipertrofia ventricular esquerda, frequentemente apresentados em pacientes renais crônicos em decorrência do aumento volêmico por dificuldades de filtração glomerular.

Outros fatores incluem a disfunção mineral causada pela disfunção renal, acumulando minerais no sangue e desencadeando processos de calcificação ectópica, sobretudo vasculares, a disfunção diastólica causada por sobrecarga de aumento de volume em dialíticos e calcificação da aorta em estágios mais avançados e a lesão renal na qual a hiperfiltração geram lesões renais se manifestando como proteinúria e posteriormente podendo chegar a uma IR.

Conclui-se, portanto, que a Insuficiência Renal se torna fator de risco para o desenvolvimento da Insuficiência Cardíaca, levando ao desencadear de vários fatores que juntos ou isolados culminam na sobrecarga de um dos órgãos mestres 
do corpo humano, dessa forma a partir desse entendimento abre-se a gama de conhecimentos com a sedimentação desses fatores e explanação acerca de como são desenvolvidos e atuam para o desfecho final, que seria a Insuficiência Cardíaca.

Pelo fato das enfermidades citadas possuírem alta prevalência na sociedade, fica evidente a necessidade de serem realizados mais pesquisas e trabalhos que envolvam os manejos e medidas terapêuticas dos fatores de risco, facilitando o tratamento precoce de forma adequada, para evitar o agravamento da IRC e, consequentemente, o desenvolvimento de uma insuficiência cardíaca.

\section{Referências}

Abboud, H. \& Henrich, W. L. (2010). Clinical practice. Stage IV chronic kidney disease. N Engl J Med. 362(1):56-65. 10.1056/NEJMcp0906797.

Abensur, H. (2010). Deficiência de ferro na doença renal crônica. Revista Brasileira de Hematologia e Hemoterapia. (32), 84-8. https://doi.org/10.1590/S1516-84842010005000047

Amenós, A. C., López, R. O., Pérez, J. M. P. (2017). Insuficiencia cardíaca en la enfermedad renal y déficit de hierro: importancia de la ferroterapia. Revista de la Sociedad Española de Nefrologia, 381. 10.1016/j.nefro.2017.03.027

Amenós, A. L., González-Juanatey, J. R., Gutiérrez, P. C., Gilarranz, A. M, \& Costa, C. G. (2010). Prevalencia de insuficiencia renal crónica en pacientes de alto riesgo o con enfermedad cardiovascular. Rev Esp Cardiol., 225-228. 10.1016/S0300-8932(10)70041-5.

Ammirati, A. L., Canziani, M. E. F. (2009). Fatores de risco da doença cardiovascular nos pacientes com doença renal crônica. Jornal Brasileiro de Nefrologia, 31(1), 43-48. https://www.bjnephrology.org/article/fatores-de-risco-da-doenca-cardiovascular-nos-pacientes-com-doenca-renal-cronica/

Bastos, M. G., Vieira, A. L., Pazeli J. R., Galil, A. G. (2020). Diastolic dysfunction for nephrologists: diagnosis at the point of care. Rev Assoc Med Bras, v. 66(12), p. 1750-1756. https://doi.org/10.1590/1806-9282.66.12.1750

Bortolotto, L. A. (2008). Hipertensão arterial e insuficiência renal crônica. Rev Bras Hipertens, 15(3), 152-5. http://departamentos.cardiol.br/dha/revista/153/09-hipertensao.pdf

Bucharles, S. G. E.,Varela, A. M., Barberato, S. H., \& Pecoits-Filho, R.(2010). Avaliação e manejo da doença cardiovascular em pacientes com doença renal crônica. Jornal Brasileiro de Nefrologia, 32(1), 120-127. https://doi.org/10.1590/S0101-28002010000100019

Canziani, M. E. F. (2004). Doenças Cardiovasculares na Doença Renal Crônica. Jornal Brasileiro de Nefrologia, XXVI(3). https://bjnephrology.org/wpcontent/uploads/2019/11/jbn_v26n3s1a08.pdf

Denic, A., Mathew, J., \& Lerman, L. O., et al. (2017). Single-Nephron Glomerular Filtration Rate In Healthy Adults. $N$ Engl J Med, 376-2349. 10.1056/NEJMoa1614329

Dizeo, C. et al. (2012). Síndrome cardiorrenal como predictor de mala evolución intrahospitalaria en pacientes añosos internados con insuficiencia cardíaca. Insuf. card., Ciudad Autónoma de Buenos Aires, 7(3), 102-108. https://www.redalyc.org/articulo.oa?id=321927799002

Dummer, C. D., Thome, F., S., \& Veronese, F. V. (2007). Doença renal crônica crônica, inflamação e aterosclerose: novos conceitos de um velho problema. Rev Assoc Med Bras., 53(5), 446-450. https://www.lume.ufrgs.br/bitstream/handle/10183/67345/000716178.pdf?sequence=1

Guimarães, L. R. M., Ferreira, A. A., \& Leonichely R. M. (2010). V Mostra Interna de Trabalhos de Iniciação Científica, CESUMAR - Centro Universitário de Maringá - Paraná. http://www.cesumar.br/prppge/pesquisa/mostras/quin_mostra/leonichely_rodrigues_macario_guimaraes.pdf

Kumar, V., Abbas, A. K.; Fausto, N., \& Mitchell, R. N. (2013). Robbins. Patologia básica. (9a ed.), 366-541. Elsevier.

Kusumota, L., Rodrigues, R. A. P., \& Marques, S. (2004). Idosos com insuficiência renal crônica: alterações do estado de saúde. Rev. Latino-Am. Enfermagem, 12(3), 525-532. https://doi.org/10.1590/S0104-11692004000300011

Lucca, L. J., Lobão, R. R. S., \& Kharol, C. (2011). Concentração de Cálcio no Dialisado e Hipercalemia na DRC. Jornal Brasileiro de Nefrologia, 33. https://www.scielo.br/j/jbn/a/TkvxGdS5TdzzgCpTcf6pdFs/?format=pdf\&lang=pt

Martins, H., Pedro, N., Castellano, M., Monteiro, P., Moura, J. J., Providência, L. H. (2011). Síndrome Cardiorrenal, os desafios no tratamento da insuficiência cardíaca. Serviços de Cardiologia e de Medicina Interna. Hospitais da Universidade de Coimbra. Coimbra. Acta Med Port, 24: $285-292$. https://pubmed.ncbi.nlm.nih.gov/22011601/

Pecoits-Filho, R., Stevinkel, P., Lindholm, B., Bergstrõm, J., Noronha, I., \& Abensur, H. (2002). Revisão: Desnutrição, inflamação e aterosclerose (síndrome MIA) em pacientes portadores de insuficiência renal crônica. Jornal Brasileiro de Nefrologia, 24(3), 136-46. https://bjnephrology.org/wpcontent/uploads/2019/11/jbn_v24n3a03.pdf

Perkovic, V., Jardine, M. J., Neal, B., et al. (2009). Canagliflozin and Renal Outccomes in Type 2 Diabetes and Nephropathy. N Engl J Med, 380:2295. 10.1056/NEJMoa1811744

Porto, R., A., Truit, M. R., Bucharlis, S. E. G., \& Hauser, A. B. (2016). Hiperparatireoidismo secundário: uma complicação da Doença Renal Crônica. Revista $R B A C$, [s. 1.], n. 80210-170. http://www.rbac.org.br/artigos/hiperparatireoidismo-secundario-uma-complicacao-da-doenca-renal-cronica-48n-3/ 
Research, Society and Development, v. 10, n. 13, e422101321496, 2021

(CC BY 4.0) | ISSN 2525-3409 | DOI: http://dx.doi.org/10.33448/rsd-v10i13.21496

Romero-González, G. et al. (2020) Burden and challenges of heart failure in patients with chronic kidney disease. A call to action. Nefrología (English Edition), 40(3), 223-236. 10.1016/j.nefro.2019.10.005

Souza, M. T., Silva, M. D., \& Carvalho, R. (2010). Revisão integrativa: o que é e como fazer. Einstein. 8(1), 102-106. https://www.scielo.br/j/eins/a/ZQTBkV $\mathrm{JZqcWrTT} 34 \mathrm{cXLjtBx} /$ ?format=pdf\&lang=pt

Truyst, C., Pecoit-Filho, M., Moraes, T. P., \& Jorgetti, V. (2021). Mortalidade cardiovascular em diálise peritoneal: o impacto dos distúrbios minerais. Jornal Brasileiro de Nefrologia, 43, 182-90. https://doi.org/10.1590/2175-8239-JBN-2020-0040

Vasan, R., Larson, M., Benjamin, E., Evans, J., Reiss, C., \& Levy, D. (1999). Congestive heart failure in subjects with normal versus reduced left ventricular ejection fraction: Prevalence and mortality in a population-based cohort. Journal of the American College of Cardiology. 10.1016/s0735-1097(99)00118-7 